\title{
Thin Circular Disk Shells of OH Masers Toward SNRs
}

\author{
Yu Zhi-yao \\ Shanghai Astronomical Observatory, 80 Nandan Road, Shanghai \\ 200030, China \\ National Astronomical Observatories, Chinese Academy of Sciences, \\ China
}

\begin{abstract}
We propose a new model of distinct thin circular disk shells to analyze Gaussian fits to spectral features of $\mathrm{OH}$ emission at $1720 \mathrm{MHz}$ toward supernova remnants. We have inferred that the $\mathrm{OH}(1720 \mathrm{MHz})$ maser features toward G348.5+0.1 are radiated from three distinct thin circular disk shells, and have obtained the parameters of the shells.
\end{abstract}

\section{Introduction}

Here we propose a new model of distinct thin circular disk shells to analyze the Gaussian fits to spectral features of $\mathrm{OH}$ emission at $1720 \mathrm{MHz}$ toward the supernova remnant G348.5+0.1 (CTB 37A).

\section{Theory and Results}

The detection of $\mathrm{OH}(1720 \mathrm{MHz})$ from G348.5+0.1 by Frail et al. (1996) is plotted in Figure 4 and Gaussian fits to the 10 spectral features are listed in Table 2 of their paper. Their figure is a map of the 10 features projected on the sky. After further analysis we can obtain with Figure 4 of their paper that the shell-like structure of SNRs suggests a new model of distinct thin circular disk shells, which explains the distribution of the 10 spectral features around G348.5+0.1. Assume that the center of G348.5+0.1 is at a point 0 at the coordinate-axes of Figure 4 of Frail et al. (1996). Its coordinates are (R.A.) $)_{0}$ and (Decl. $)_{0}$. According to Table 2 of Frail et al. (1996), we obtain the (R.A.) $i$ and (Decl. $)_{i}$ of i-th maser feature. Then the differences of the coordinates between the center and the $\mathrm{i}$-th radio source are $\Delta$ (R.A. $)_{i}=(\text { R.A. })_{i}-(\text { R.A. })_{0}$ and $\Delta(\text { Decl. })_{i}=(\text { Decl. })_{i}-(\text { Decl. })_{0}$. For a thin circular disk shell its radius is defined. If a thin circular disk shell is face-on, the projection of the shell is circular. If a circular disk shell is edge-on, the projection of the shell is linear. If a thin circular disk shell is neither face-on nor edge-on, the projection of the shell is an ellipse. For the maser features on a thin circular disk shell their coordinates have the following relationship:

$$
\frac{\left[\Delta(\text { R.A. })_{i}\right]^{2}}{a^{2}}+\frac{\left[\Delta(\text { Decl. })_{i}\right]^{2}}{b^{2}}=1
$$


From Eq. (1) we can obtain

$$
\left[\Delta(\text { Decl. })_{i}\right]^{2}=\rho^{2}-\frac{\left[\Delta(\text { R.A. })_{i}\right]^{2}}{\cos ^{2} \theta},
$$

where $\rho$ is the radius of the shell and $\theta$ is the inclination angle between the line of sight and the normal of the circular disk-shell. For a face-on thin circular disk shell, $\rho=a=b, \theta=0$, and $\cos \theta=1$, i.e. $\left.\left[\Delta(\text { Decl. })_{i}\right]^{2}=\rho^{2}-[\Delta \text { (R.A. })_{i}\right]^{2}$. For an edge-on thin circular disk shell, $\Delta$ (R.A. $)_{i}=C$ or $\Delta(\text { Decl. })_{i}=C$, or $\frac{\Delta(\text { Decl. })_{i}}{\Delta \text { (R.A. })_{i}}=C$, where $C$ is a constant. For a non-face-on or non-edge-on thin circular disk shell, $a \neq b$. If $b>a, \rho=b, \cos \theta=a / b, \theta=\cos ^{-1}(a / b)$. If $a>b, \rho=a, \cos \theta=b / a, \theta=\cos ^{-1}(b / a)$. From Eq. (2) we obtain that for a particular thin circular disk shell both $a$ and $b$ are defined, and the coordinates of the maser features distributed on the thin circular disk shell, $\Delta(\text { Decl. })_{i}$ and $\Delta$ (R.A. $)_{i}$, need to satisfy Eq. (2). We can see from Eq. (2) that a thin circular disk shell has its particular radius $\rho$ and inclination angle $\theta$. For each shell, $\left[\Delta(\text { Decl. })_{i}\right]^{2}$ is a monotonically decreasing function of $\left[\Delta(\text { R.A. })_{i}\right]^{2}$. Thus we can divide the thin circular disk shell-like structure according to those regions where the distribution of points is arranged following a monotonically decrease of $\left[\Delta(\text { Decl. })_{i}\right]^{2}$ vs. $\left.[\Delta \text { (R.A. })_{i}\right]^{2}$. We can then do a statistical fit. We select several different centers to do the statistical fitting according to the mentioned steps, until the error of the fit is a minimum. We can obtain that the 10 maser features around G348.5+0.1 are distributed on three distinct thin circular disk shells. Finally, we take the center coordinates of G348.5+0.1 as R.A. (1950) = $17^{\mathrm{h}} 11^{\mathrm{m}} 05^{\mathrm{s}} .0$ and Decl. $(1950)=-38^{\circ} 30^{\prime} 00^{\prime \prime}$ from Frail et al. (1996). The values of $\left[\Delta(\text { R.A. })_{i}\right]^{2}$ and $\left[\Delta(\text { Decl. })_{i}\right]^{2}$ of the 10 maser features are obtained from Table 2 of their paper. We obtain that the 10 maser features are distributed on three distinct thin circular disk shells, with corresponding $\rho$ (mas) of $154.7,261.8$, and 509.0 , and $\theta$ of $51.5,38^{\circ} 2$, and $38^{\circ} .1$, respectively. Our results indicate that the shell-like structure is made up of several thin circular disk shells. These results are important for the investigation of radio morphology of SNRs.

Acknowledgments. This work was supported by the National Natural Sciences Foundation of China.

\section{References}

Frail, D. A., Goss, W. M., Reynoso, E. M., Giacani, E. B., Green, A. J., \& Otrupcek, R. 1996, AJ, 111, 1651 